\title{
Development of Optical Fiber Fence Alarm System with Video Linkage
}

\section{Function}

\author{
Yuan Qinghui ${ }^{1, a,{ }^{*},}$ Nie Xiujun ${ }^{1, b}$ and Feng Guofang ${ }^{2, c}$ \\ ${ }^{1}$ Binzhou Polytechnic, Binzhou, Shandong Province, 256603 \\ ${ }^{2}$ Sixth Primary Schools in Bincheng, Binzhou, Shandong Province,256600 \\ asdyqh1979@126.com, b443183538@qq.com, c48908892@qq.com
}

Keywords: Optical fiber fence, Video monitoring, Sagnac interference

\begin{abstract}
A new visualized fiber fence alarm system was developed in this paper, which used Sagnac interferometric sensor of optical fiber fence in the surrounding invasion signal detection and positioning, and then sent alarm information and transfer video signals to the control center, so that the monitoring could switch to intrusion scene timely. This visualized alarm system makes full use of advantages of optical fiber detection and video surveillance, which improved the level of intelligence of security system and the security performance of the system.
\end{abstract}

\section{Introduction}

In this highly developed technological society, we urgently need to strengthen the security of modern technology to face the diversified criminal means. In a sense, the security technology is a new technology which combined with electronic technology, computer technology, modern communication technology, sensor technology and other high-techs, which plays an important role in preventing crime, maintaining public order and protecting people and property safety ${ }^{[1 \sim 4]}$.

Such as infrared radiation, microwaves, leaked cables and electronic fence, the traditional security technology and security alarm system has made a contribution in security technology. However, there are some flaws (objective technical conditions) to restrict this development. For example, active infrared fence alarm system limited by the terrain conditions. Leaked cables, electronic fences and other fence alarm system have a large power consumption. Moreover, they are susceptible to electromagnetic interference, signal interference and crosstalk. Compared to these traditional alarm system, new security fence alarm system has remarkable advantages, such as anti-electromagnetic interference, electrical insulation, low transport energy consumption, high reliability, remarkable positioning capability. This new sensing technologies include optical time domain reflectometer positioning technology, monitoring the invasion by Sagnac interferometer and monitoring and positioning reflection wavelength by fiber bragg grating (FBG) ${ }^{[5]}$. Based on the Sagnac interferometer, these fiber optic sensors have outstanding advantages of effectively, easily installing, easily maintenance and low cost in these technologies. In this paper, we introduced a fence intrusion detection security system, which based on Sagnac interferometric fiber optic sensors. Finally, the application was tested ${ }^{[6 \sim 9]}$.

\section{Fiber Fence Alarm Security System Based on Sagnac Interferometric Sensor}

(1)Principle of Optical Fiber Sagnac Interferometric.

The structure of optical fiber Sagnac interferometric was shown in Fig.1. When a light emitted from the light source, it was divided into the same two beams of light and coupled into the same 
optical fiber loop. Two formed coherent lights, which own the opposite directions, were converged in the coupler. The interference would occur in the coupler when these two lights were under the condition of same frequency interference, vibration direction and the constant phase difference. Finally, the interference phenomenon trended to be stabilized when the sensing fiber was not disturbed by the interference.

When the system was subjected to external interference, it would produce different phase shifts and the optical detector could detect this change. Suppose A was a fiber produced weak vibration around the intruder, the interference was occurred when they returned to the start of convergence and the phase difference between the two beams of light from the optical fiber transmission could be detected. Combined with signal processing and mathematical physics method, we finally obtained the information of optical signal intrusion event location and alerts.

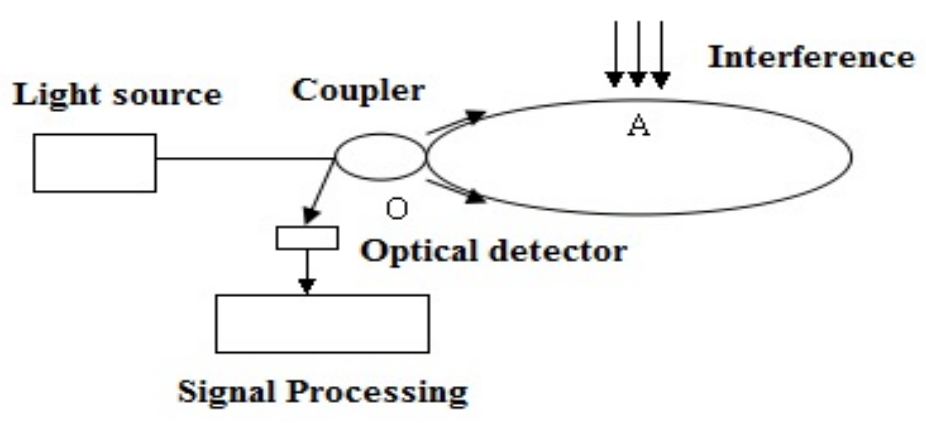

Fig.1 The structure of optical fiber Sagnac interferometric

\section{(2)Principle of System.}

This system consists of a central controller, signal processing, sensor, sensor end isolation, transmission and sensor cables and other components, which was shown in Fig.2. The terminal may generate the signal processing, convert into the sensor information and transmit via conduction cables. Cable isolator is located between the sensor isolation and sensor terminator and laid in the area in need of protection. The vibration characteristics of the various external intrusion sensor cable from the changes caused by the optical signal transmission, and the signal is received by the optical signal processing terminal when detected intrusions.

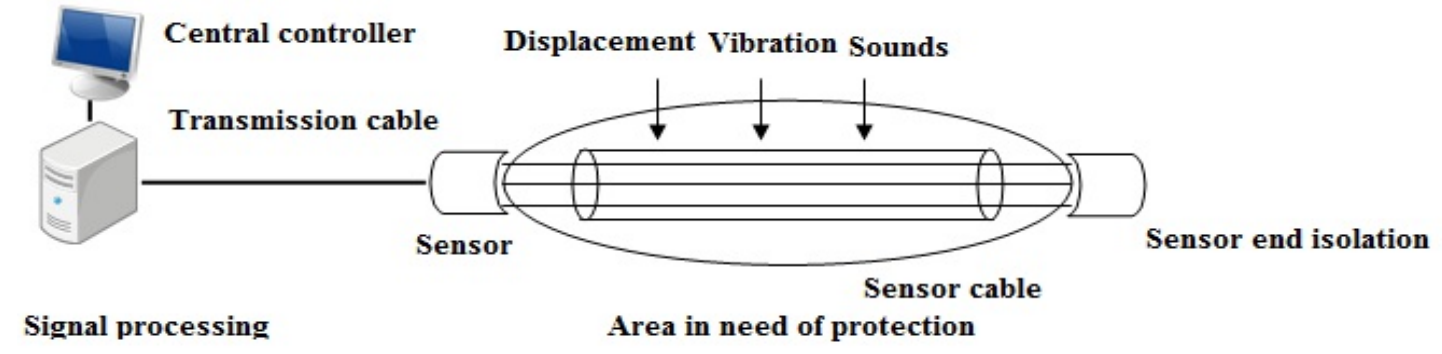

Fig. 2 The structure of system protection

\section{(3)Optical fiber alarm system software.}

Optical fiber alarm monitoring system can be divided into four parts, i.e. data collection and transmission, data processing, information management and alarm ${ }^{[10]}$.

Data collection and Transmission: a TCP link with SI 425 and read data by using socket mode, then the data transformed to sensor information by decoding method. The real-time saving and alarm data stored function of wavelength data were accomplished.

Data processing: Classify the obtained data and then the characteristic signals were extracted and analyzed. Finally, the work of data processing and alarm was accomplished.

Information management: Record probe position information and alarm log. When intrusion 
occurs, we can check the alarm position sensor and the correspondingly system information. Meanwhile, the achieved data was collected for the next check.

Alarm: Process the invasion signal timely and judge whether it is an alarm or not. If this similar warning information was recorded, no alarm response was occurred. Otherwise this system respond the alarm and check the database, finally we obtained the alarm sensor location. Based on these four parts of this system, a complete alarm function was accomplished.

\section{Video linkage system}

Based on the design of fiber fence intrusion software monitor system, perimeter security system has the accurate intrusion, video monitoring and linkage function. So we shall visualize the perimeter security and improve the ability of obtain information. As an important part in this whole system, video linkage system was divided into three parts, collection, information transmission and information processing and image display.

(1)Collection.

Part of information was collected by image collection unit and compression storage collection unit, which was located in front part of the system. Video signal was obtained by camera, and transmitted through wire or wireless transmission to the video server or hard disk recorder, then compressed and coded the video signal. Image collection and transmission unit signals were divided into wired and wireless signal transmission. In the state of prone position change monitoring, we should take the method of wireless signal transmission. The wired signal transmission mode, main equipment (camera, video server) and the transmission medium were used video cable and optical fiber to achieve wired transmission. The wireless signal transmission equipments should follow the standard IEEE 8.2.11 a/b/g/n, the structure of this part was shown in Fig.3.

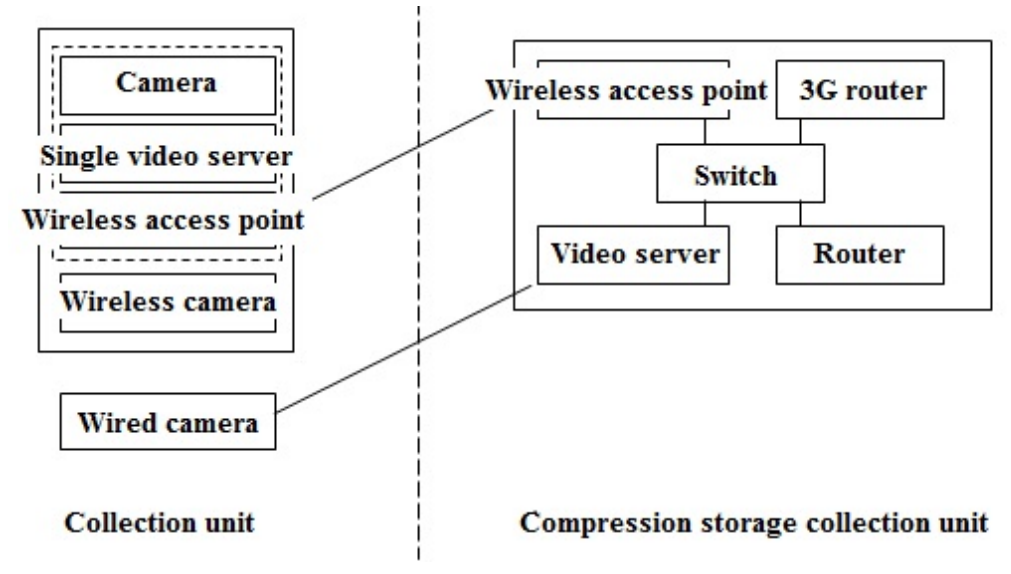

Fig.3 The structure of part of collection in video linkage system

\section{(2)Information transmission.}

The part of information transmission was the network transmission site and off-side monitoring center. The monitoring center could access and manage the video server and DVR via a linked remote monitoring center. The method of transmission signal was divided into wired and wireless signal transmission. For a cable network access or serious radio signal interference phenomenon, it should use a wired signal transmission mode, or the wireless signal transmission mode should be chosen. The structure of this part was shown in Fig.4. 


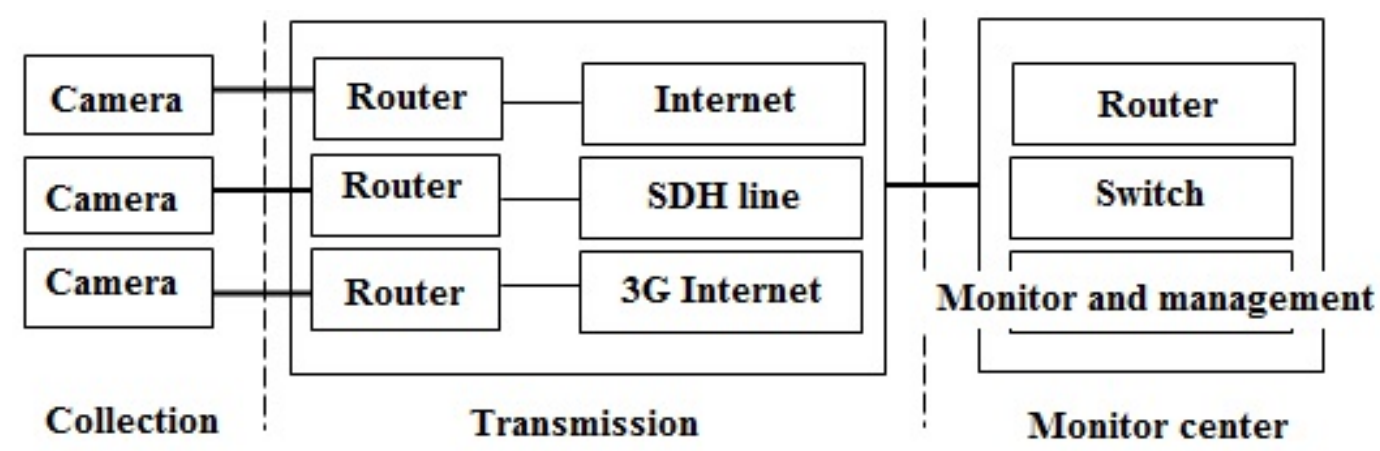

Fig.4 The structure of information transmission

(3)Information processing and image display.

Information processing and image display was located $\mathrm{n}$ the monitor center which was composed by information processing unit and image display unit. The video and digital signal was processed by a digital video decoder software and finally displayed in the video. The structure of this part was shown in Fig.5.

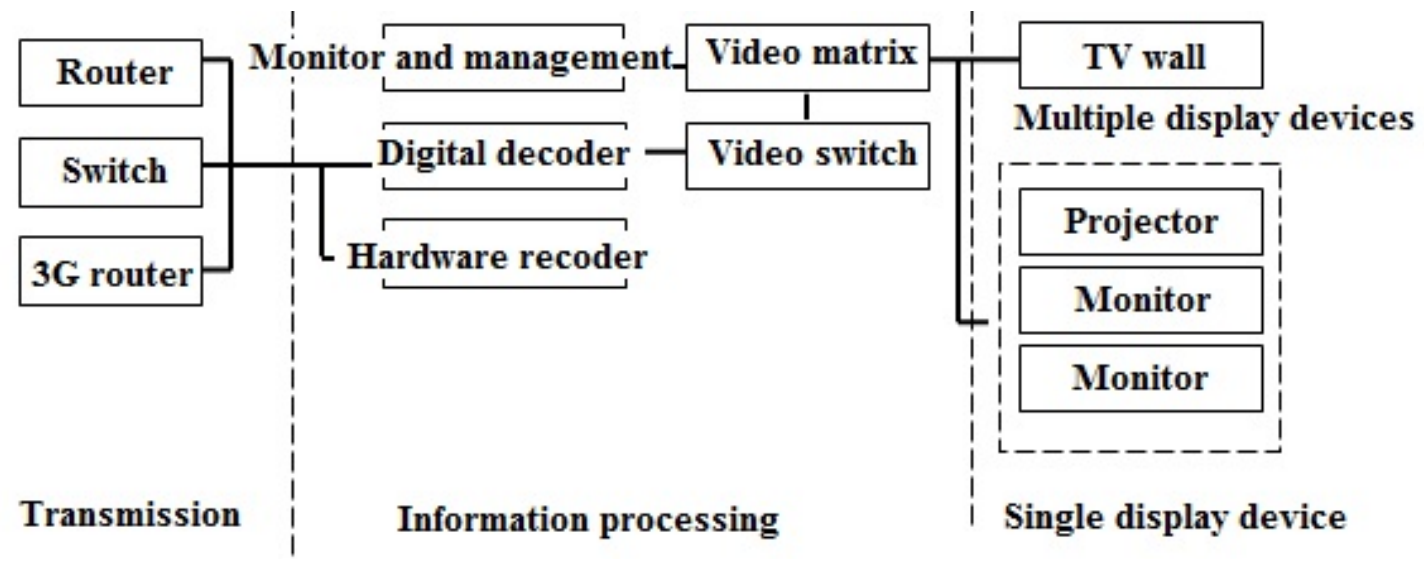

Fig.5 The structure of information processing and image display

\section{Results}

The visualization fiber fence security alarm for monitoring was applied in a district. In this application, the video monitoring system has a integrated video independent district scale optical fiber fence protection system and two hundred point monitoring, which used the same monitoring information and database. When an intrusion signal invaded, the output signal from optical fiber fence system would be destroyed. Combined with location information of the electronic point diagram, a alarm zone would be separated and system center would sent the alarm information. Further processing would be processed by the inspection person. A little 3-D intelligent pattern recognition formatted by the application of relationship among people and objects. This application was used in this district for about 1 year, and it worked stable and reliable operation, which had no false alarm event.

\section{Conclusions}

An optical fiber fence alarm system, which based on Sagnac, was introduced in this paper. This system made full use of the Sagnac optical fiber interferometer principle. When a external intrusion signal invaded, the light interference will be changed, which caused the intensity mutation of light and alarm ${ }^{[11]}$. This system realized the linkage function and integration between intrusion detection 
system and video. Not only can effectively perceive the intrusion, this linkage function also has the ability of video monitoring, intrusion forensics capability and target recognition. This system has a very low false alarm rate. This application has a broad apply prospect, which can be used in he high-grade communities, bank, airports, borders, military bases and other important facilities.

\section{Acknowledges}

The authors would like to express appreciation for the financial support by the (Binzhou Vocational College (2013XYKT13).

\section{References}

[1] I. Cernak and L.J. Noble-Haeusslein: Journal of Cerebral Blood Flow \& Metabolism Vol. 30 (2010), p. 255.

[2] B. Zhao, H.G. Zhang, J. Li, L. Chen and S. Wen: Chinese Journal of Computers Vol. 1 (2010), p. 82.

[3] A.R. Metke and R.L. Ekl: Innovative Smart Grid Technologies Vol. 1 (2010), p. 1.

[4] A.R. Metke and R.L. Ekl: Smart Grid, IEEE Transaction on Vol. 1 (2010), p. 99.

[5] B. Liu, Y.F. Yang and W.C. Niu: Photon Technology Vol. 4 (2004), p. 208.

[6] S.M. Li, S.X. Qian, L.J. Kong, Z.C. Ren, Y. Li, C. Tu and H.T. Wang: Europhysics Letters Vol. 105 (2014), p. 105.

[7] A. Bhattachryya, D.K. Gayen and T. Chattopadhyay: Optics Communications Vol. 293 (2013), p. 31.

[8] P.V.N. Kishore, M.S. Shankar, P. Kishore, P.V. Rao and S. Roy: Microwave and Photonics Vol. 1 (2013), p. 1.

[9] D. Sven, C. Carsten, F. Carsten and G. Petra: Journal of Chemical Physics, Vol. 137 (2012), p. 174201.

[10] C. Tang, X.L. Lu and H.J. Wu: Security and Safety Technology Magazine, Vol. 9 (2010), p. 20.

[11] L.X. Wang, Q. Luo and X.G. Liu: System and Research, Vol. 4 (2007), p. 60. 